\title{
CONVEX BODIES WITH FEW FACES
}

\author{
KEITH BALL AND ALAIN PAJOR
}

(Communicated by William J. Davis)

ABSTRACT. It is proved that if $u_{1}, \ldots, u_{n}$ are vectors in $\mathbf{R}^{k}, k \leq n, 1 \leq$ $p<\infty$ and

$$
r=\left(\frac{1}{k} \sum_{1}^{n}\left|u_{i}\right|^{p}\right)^{1 / p}
$$

then the volume of the symmetric convex body whose boundary functionals are $\pm u_{1}, \ldots, \pm u_{n}$, is bounded from below as

$$
\mid\left.\left\{x \in \mathbf{R}^{k}:\left|\left\langle x, u_{i}\right\rangle\right| \leq 1 \text { for every } i\right\}\right|^{1 / k} \geq 1 / \sqrt{\rho} r .
$$

An application to number theory is stated.

\section{INTRODUCTION}

In [V], Vaaler proved that if $Q_{n}=\left[-\frac{1}{2}, \frac{1}{2}\right]^{n}$ is the central unit cube in $\mathbf{R}^{n}$ and $U$ is a subspace of $\mathbf{R}^{n}$ then the volume $\left|U \cap Q_{n}\right|$, of the section of $Q_{n}$ by $U$ is at least 1 . This result may be reformulated as follows: if $u_{1}, \ldots, u_{n}$ are vectors in $\mathbf{R}^{k}, 1 \leq k \leq n$ whose Euclidean lengths satisfy $\sum_{1}^{n}\left|u_{i}\right|^{2} \leq k$ then

$$
\mid\left.\left\{x \in \mathbf{R}^{k}:\left|\left\langle x, u_{i}\right\rangle\right| \leq 1 \text { for every } i\right\}\right|^{1 / k} \geq 2 \text {. }
$$

A related theorem, (Theorem 1, below) in which the condition $\sum\left|u_{i}\right|^{2} \leq k$ is replaced by $\max _{i}\left|u_{i}\right| \leq 1$ was proved by Carl and Pajor [CP] and Gluskin [G]. Gluskin's methods enable him to obtain sharp results in limiting cases which in turn have applications in harmonic analysis. Results closely related to Theorem 1 were also obtained by Bárány and Füredi [BF] and Bourgain, Lindenstrauss and Milman [BLM].

Theorem 1. There is a constant $\delta>0$ so that if $u_{1}, \ldots, u_{n} \in \mathbf{R}^{k}, 1 \leq k \leq n$ are vectors of length at most 1 then

$$
\mid\left.\left\{x \in \mathbf{R}^{k}:\left|\left\langle x, u_{i}\right\rangle\right| \leq 1 \text { for every } i\right\}\right|^{1 / k} \geq \delta / \sqrt{1+\log (n / k)} .
$$

The estimate is best possible if $n$ is at most exponential in $k$, apart from the value of the constant $\delta$. This is demonstrated by an example which had

Received by the editors September 14, 1989.

1980 Mathematics Subject Classification (1985 Revision). Primary 52A20, 10 E05.

The first author was partially supported by N. S. F. DMS- 8807243 .

(C) 1990 American Mathematical Society $0002-9939 / 90 \$ 1.00+\$ .25$ per page 
appeared some time earlier in a paper of Figiel and Johnson [FJ]. Theorem 1 gives a lower bound on the volume ratios of the unit balls of $k$-dimensional subspaces of $l_{\infty}^{n}$ and hence on the distance of these subspaces from Euclidean space.

Regarding Theorem 1 as a $p=\infty$ version of Vaaler's $p=2$ result, Kashin asked whether a similar result holds for $2<p<\infty$. This question is answered in the affirmative by the following theorem.

Theorem 2. Suppose $u_{1}, \ldots, u_{n} \in \mathbf{R}^{k}$ with $k \leq n, 1 \leq p<\infty$ and let

$$
r=\left(\frac{1}{k} \sum_{1}^{n}\left|u_{i}\right|^{p}\right)^{1 / p} .
$$

Then

$$
\mid\left.\left\{x \in \mathbf{R}^{k}:\left|\left\langle x, u_{i}\right\rangle\right| \leq 1 \text { for every } i\right\}\right|^{1 / k} \geq \begin{cases}2 \sqrt{2} / \sqrt{p} r & \text { if } p \geq 2 \\ 1 / r & \text { if } 1 \leq p \leq 2 .\end{cases}
$$

The lower bound is best possible (up to a constant) provided $e^{p} k \leq n \leq e^{k}$.

Remark. The slightly stronger result for $p \geq 2$ is isolated since for $p=2$ it gives back exactly Vaaler's result.

Theorem 1 follows immediately from Theorem 2 by a standard optimization argument. If $\left(u_{i}\right)_{1}^{n}$ in $\mathbf{R}^{k}$ all have norm at most 1 then for any $p \in[1, \infty)$,

$$
\left(\frac{1}{k} \sum_{k}^{n}\left|u_{i}\right|^{p}\right)^{1 / p} \leq\left(\frac{n}{k}\right)^{1 / p}
$$

so that

$$
\mid\left.\left\{x:\left|\left\langle x, u_{i}\right\rangle\right| \leq 1 \text { for every } i\right\}\right|^{1 / k} \geq 2 \sqrt{2} / \sqrt{p}\left(\frac{n}{k}\right)^{1 / p}
$$

(for $p \geq 2$ ) and the latter is at least $2 / \sqrt{e} \sqrt{1+\log (n / k)}$ when $p=$ $2(1+\log (n / k))$.

With the careful use of well-known methods for estimating the entropy of convex bodies it is possible to obtain more general (but less precise) estimates than that provided by Theorem 2; (see [BP]). The purpose of this paper is to provide a very short proof of Theorem 2 , and a fortiori, Theorem 1 .

Vaaler originally proved his theorem because of its applications to the geometry of numbers. The last section of this paper includes a statement of the generalization of Siegel's lemma which follows from Theorem 2.

\section{THE LOWER BOUND}

The proof of Theorem 2 makes use of the following result from [MeP] which was designed to extend Vaaler's theorem in a different direction: it estimates 
the volumes of sections of the unit balls of the spaces $l_{p}^{n}, 1 \leq p \leq \infty$. For $1 \leq p \leq \infty, n \in \mathbf{N}$ let

$$
B_{p}^{n}=\left\{x \in \mathbf{R}^{n}: \sum_{1}^{n}\left|x_{i}\right|^{p} \leq 1\right\}
$$

be the unit ball of $l_{p}^{n}$.

Theorem 3. Let $U$ be a $k$-dimensional subspace of $\mathbf{R}^{n}$; if $1 \leq p \leq q \leq \infty$ then

$$
\frac{\left|B_{p}^{n} \cap U\right|}{\left|B_{p}^{k}\right|} \leq \frac{\left|B_{q}^{n} \cap U\right|}{\left|B_{q}^{k}\right|}
$$

Remark. The case $p=2, q=\infty$ is Vaaler's theorem since then, the left side is 1 and the inequality states that

$$
\left|B_{\infty}^{n} \cap U\right| \geq\left|B_{\infty}^{k}\right| \geq 2^{k} .
$$

For notational convenience, the proof of Theorem 2 is divided into several short lemmas. The first is no more than a convenient form of Hölder's inequality. For $k \in \mathbf{N}, S^{k-1}$ will denote the Euclidean sphere in $\mathbf{R}^{k}$ and $\sigma=\sigma_{k-1}$, the rotationally invariant probability measure on $S^{k-1}$. Also let $v_{k}$ be the volume of the Euclidean unit ball in $\mathbf{R}^{k}$.

Lemma 4. Let $C$ and $B$ be symmetric convex bodies in $\mathbf{R}^{k}$ with Minkowski gauges $\|\cdot\|_{C}$ and $\|\cdot\|_{B}$, respectively. Then for $p>0$

$$
\left(\frac{|C|}{|B|}\right)^{1 / k} \geq\left(\frac{k+p}{k|B|} \int_{B}\|x\|_{C}^{p} d x\right)^{-1 / p} \text {. }
$$

Proof.

$$
\begin{aligned}
\left(\frac{|C|}{|B|}\right)^{1 / k} & =\left(\frac{v_{k}}{|B|} \int_{S^{k-1}}\|\theta\|_{C}^{-k} d \sigma(\theta)\right)^{1 / k} \\
& =\left(\frac{k v_{k}}{|B|} \int_{S^{k-1}}\left(\frac{\|\theta\|_{B}}{\|\theta\|_{C}}\right)^{k} \int_{0}^{\|\theta\|_{B}^{-1}} r^{k-1} d r d \sigma(\theta)\right)^{1 / k} \\
& =\left(\frac{1}{|B|} \int_{B}\left(\frac{\|x\|_{B}}{\|x\|_{C}}\right)^{k} d x\right)^{1 / k} \\
& \geq\left(\frac{1}{|B|} \int_{B}\left(\frac{\|x\|_{B}}{\|x\|_{C}}\right)^{-p} d x\right)^{-1 / p} \\
& =\left(\frac{k+p}{k|B|} \int_{B}\|x\|_{C}^{p} d x\right)^{-1 / p} \cdot
\end{aligned}
$$

(Lemma 4 appears in [MiP] as Corollary 2.2.) 
Lemma 5. Suppose $u_{1}, \ldots, u_{n} \in \mathbf{R}^{k}$ with $k \leq n$ and $1 \leq p<\infty$. Then

$$
\begin{aligned}
\mid\{x & \left.\in \mathbf{R}^{k}:\left|\left\langle x_{i}, u_{i}\right\rangle\right| \leq 1 \text { for every } i\right\}\left.\right|^{1 / k} \\
& \geq 2\left(\frac{k+p}{k} \sum_{i=1}^{n} \frac{1}{\left|B_{p}^{k}\right|} \int_{B_{p}^{k}}\left|\left\langle x, u_{i}\right\rangle\right|^{p} d x\right)^{-1 / p} .
\end{aligned}
$$

Proof. Define $T: \mathbf{R}^{k} \rightarrow \mathbf{R}^{n}$ by $(T x)_{i}=\left\langle x, u_{i}\right\rangle, 1 \leq i \leq n$ and let $U=T\left(\mathbf{R}^{k}\right)$. The problem is to estimate from below

$$
\left|T^{-1}\left(B_{\infty}^{n}\right)\right|^{1 / k}=\left|T^{-1}\left(U \cap B_{\infty}^{n}\right)\right|^{1 / k} .
$$

By Theorem 3,

$$
\left|U \cap B_{\infty}^{n}\right|^{1 / k} \geq 2\left(\frac{\left|U \cap B_{p}^{n}\right|}{\left|B_{p}^{k}\right|}\right)^{1 / k}
$$

and so

$$
\left|T^{-1}\left(B_{\infty}^{n}\right)\right|^{1 / k} \geq 2\left(\frac{\left|T^{-1}\left(B_{p}^{n}\right)\right|}{\left|B_{p}^{k}\right|}\right)^{1 / k} .
$$

Regard $T$ as an operator: $l_{p}^{k} \rightarrow l_{p}^{n}$. Then by Lemma 4,

$$
\begin{aligned}
2\left(\frac{\left|T^{-1}\left(B_{p}^{n}\right)\right|}{\mid B_{p}^{k}}\right)^{1 / k} & \geq 2\left(\frac{k+p}{k\left|B_{p}^{k}\right|} \int_{B_{p}^{k}}\|T x\|^{p} d x\right)^{-1 / p} \\
& =2\left(\frac{k+p}{k\left|B_{p}^{k}\right|} \int_{B_{p}^{k}} \sum_{1}^{n}\left|\left\langle x, u_{i}\right\rangle\right|^{p} d x\right)^{-1 / p} .
\end{aligned}
$$

Proof of Theorem 2. Let $\left(u_{i}\right)_{1}^{n}$ and $p$ be as above. For each $i$ let $v_{i}$ be the unit vector in the direction of $u_{i}$. By Lemma 5 ,

$$
\begin{aligned}
\mid\{x & \left.\in \mathbf{R}^{k}:\left|\left\langle x, u_{i}\right\rangle\right| \leq 1 \text { for every } i\right\}\left.\right|^{1 / k} \\
& \geq 2\left(\frac{k+p}{k} \sum_{i=1}^{n} \frac{1}{\left|B_{p}^{k}\right|} \int_{B_{p}^{k}}\left|\left\langle x, u_{i}\right\rangle\right|^{p} d x\right)^{-1 / p} \\
& =2\left(\frac{k+p}{k} \sum_{1}^{n}\left|u_{i}\right|^{p} \cdot \frac{1}{\left|B_{p}^{k}\right|} \int_{B_{p}^{k}}\left|\left\langle x, v_{i}\right\rangle\right|^{p} d x\right)^{-1 / p} \\
& \geq 2\left(\frac{1}{k} \sum_{i=1}^{n}\left|u_{i}\right|^{p}\right)^{-1 / p} \min \left(\frac{k+p}{\left|B_{p}^{k}\right|} \int_{B_{p}^{k}}|\langle x, v\rangle|^{p} d x\right)^{-1 / p}
\end{aligned}
$$

where the minimum is taken over all vectors $v$ of Euclidean length 1. So to complete the proof it suffices to show that for such a vector $v$,

$$
\left(\frac{k+p}{\left|B_{p}^{k}\right|} \int_{B_{p}^{k}}|\langle x, v\rangle|^{p} d x\right)^{1 / p} \leq \begin{cases}\sqrt{p / 2} & \text { if } p \geq 2 \\ 2 & \text { if } 1 \leq p<2\end{cases}
$$


Let $\left(x^{(j)}\right)_{1}^{k}$ and $\left(v^{(j)}\right)_{1}^{k}$ be the coordinates of the vectors $x$ and $v$ in $\mathbf{R}^{k}$. For $p \geq 2$, observe that the functions $\left(x^{(j)} v^{(j)}\right)$ on $B_{p}^{k}$ form a conditionally symmetric sequence, so by Khintchine's inequality and Hölder's inequality (for $\left.\sum_{1}^{n} v^{(j) 2}=1\right)$,

$$
\begin{aligned}
\left(\frac{k+p}{\left|B_{p}^{k}\right|} \int_{B_{p}^{k}}\left|\sum_{1}^{k} x^{(j)} v^{(j)}\right|^{p} d x\right)^{1 / p} \\
\quad \leq \sqrt{\frac{p}{2}}\left(\frac{k+p}{\left|B_{p}^{k}\right|} \int_{B_{p}^{k}}\left(\sum_{1}^{k} x^{(j) 2} v^{(j) 2}\right)^{p / 2} d x\right)^{1 / p} \\
\quad \leq \sqrt{\frac{p}{2}}\left(\frac{k+p}{\left|B_{p}^{k}\right|} \int_{B_{p}^{k}} \sum_{1}^{n}\left|x^{(j)}\right|^{p} \cdot v^{(j) 2} d x\right)^{1 / p} \\
\quad=\sqrt{\frac{p}{2}}\left(\frac{k+p}{\left|B_{p}^{k}\right|} \int_{B_{p}^{k}}\left|x^{(1)}\right|^{p} d x\right)^{1 / p} \\
\quad=\sqrt{\frac{p}{2}} .
\end{aligned}
$$

For $1 \leq p<2$ it is easily checked that

$$
\begin{aligned}
& \left(\frac{k+p}{\left|B_{p}^{k}\right|} \int_{B_{p}^{k}}|\langle x, v\rangle|^{p} d x\right)^{1 / p} \\
& \leq(k+p)^{1 / p}\left(\frac{1}{\left|B_{p}^{k}\right|} \int_{B_{p}^{k}}\langle x, v\rangle^{2} d x\right)^{1 / 2} \\
& \quad=(k+p)^{1 / p}\left(\frac{1}{\left|B_{p}^{k}\right|} \int_{B_{p}^{k}}\left(x^{(1)}\right)^{2} d x\right)^{1 / 2}
\end{aligned}
$$

and the last expression can be (rather roughly) estimated by 2 using standard inequalities involving logarithmically concave functions.

Remark. The proof of Theorem 2 can be simplified even further if the integration over $B_{p}^{k}$ is replaced by integration over $S^{k-1}$ (and Hölder's inequality applied here). The proof was presented as above because Lemma 5 has some intrinsic interest: for example it may be used to recover Gluskin's precise estimate as follows. Suppose $m \in \mathbf{N}$ and the vectors $\left(z_{i}\right)_{1}^{m} \in \mathbf{R}^{k}$ satisfy

$$
\left|z_{i}\right| \leq(\log (1+m / k))^{-1 / 2}, \quad 1 \leq i \leq m .
$$

For $\varepsilon>0$, let $W(\varepsilon)$ be the set

$$
\left\{x \in \mathbf{R}^{k}: \max _{j}\left|x^{(j)}\right| \leq 1, \max _{i}\left|\left\langle x, z_{i}\right\rangle\right| \leq \frac{1}{\varepsilon}\right\}
$$


that is, $W(\varepsilon)$ is the intersection of the cube $B_{\infty}^{k}$ with $m$ "bands" of width at most $(2 / \varepsilon) \sqrt{\log (1+m / k)}$. Then $|W(\varepsilon)|^{1 / k} \rightarrow 2$ as $\varepsilon \rightarrow 0$, uniformly in $k$ and $m$. To see this, apply Lemma 5 with $n=k+m$, the first $k, u_{i}$ 's being the standard basis vectors $\mathbf{R}^{k}$ and the remaining $m$ being the vectors $\left(\varepsilon z_{i}\right)_{1}^{m}$. If $e_{j}$ is a standard basis vector,

$$
\frac{k+p}{\left|B_{p}^{k}\right|} \int_{B_{p}^{k}}\left|\left\langle x, e_{j}\right\rangle\right|^{p} d x=1
$$

and so Lemma 5 (and the proof of Theorem 2) show that for each $p \geq 2$, $|W(\varepsilon)|^{1 / k} \geq 2\left(1+m / k(p / 2)^{p / 2} \varepsilon^{p}(\log (1+m / k))^{-p / 2}\right)^{-1 / p}$ and the latter is at least $2 /(1+\sqrt{e} \cdot \varepsilon)$ if $p=\max (2,2 \log (1+m / k))$.

As was briefly mentioned earlier, more general estimates than that of Theorem 2 are obtained in [BP] (for entropy numbers instead of volumes). It is worth noting however that even the argument of Theorem 2 can be used to give the following: there is a constant $c$ so that if $u_{1}, \ldots, u_{n} \in \mathbf{R}^{k}, k \leq n$ and $T: l_{2}^{k} \rightarrow l_{\infty}^{n}$ is given by $(T x)_{i}=\left\langle u_{i}, x\right\rangle, 1 \leq i \leq n$, then the $k$ th entropy number of $T$ satisfies

$$
e_{k}(T) \leq \frac{c \sqrt{p}}{\sqrt{k}}\left(\frac{1}{k} \sum_{1}^{n}\left|u_{i}\right|^{p}\right)^{1 / p}\left(1+\log \frac{n}{k}\right)^{1 / p}
$$

and hence

$$
e_{k}(T) \leq \frac{e c}{\sqrt{k}} \sqrt{1+\log \frac{n}{k}} \cdot\|T\|
$$

(taking $p=2(1+\log (n / k))$. To obtain this, one uses Schütt's estimates [S] for the entropy numbers of the formal identity from $l_{p}^{n}$ to $l_{\infty}^{n}$ in place of the result of Meyer and Pajor, an the dual Sudakov inequality of Pajor and Tomczak, [PT] in place of the application of Hölder's inequality.

\section{AN APPLICATION TO LINEAR FORMS}

As stated in the Introduction, Vaaler's original result has applications to the geometry of numbers. One such, a sharpened form of Siegel's lemma, is given in [BV]. Using the arguments of Bombieri and Vaaler and Theorem 2, one can obtain the generalization of their result, contained in Theorem 6 , below. Some notation is needed. If $A$ is a $k \times n$ matrix of reals with independent rows $(1 \leq k \leq n)$, denote by $v_{j}=v_{j}(A), 1 \leq j \leq k$, the rows of $A$. Let $\left(e_{i}\right)_{1}^{n}$ be the standard basis of $\mathbf{R}^{n}$ and denote by $c_{i}$, the distance (in the Euclidean norm) of $e_{i}$ from the span of the $v_{j}$ 's in $\mathbf{R}^{n}$. (So if $A_{i}$ is the matrix with $k+1$ rows, $v_{1}, \ldots, v_{n}, e_{i}$ then

$$
\left.c_{i}^{2}=\frac{\operatorname{det}\left(A_{i} A_{i}^{*}\right)}{\operatorname{det}\left(A A^{*}\right)} \quad \text { for } 1 \leq i \leq n .\right)
$$


Theorem 6. Let $A$ be a $k \times n$ matrix with rank $k$ and integral entries. With the notation above, the system $A x=0$ admits $n-k$ linearly independent solutions

$$
z^{(r)}=\left(z_{1}^{(r)}, \ldots, z_{n}^{(r)}\right) \in \mathbf{Z}^{n}, \quad 1 \leq r \leq n-k
$$

so that for every $p \geq 2$,

$$
\prod_{1 \leq r \leq n-k} \max _{i}\left|z_{i}^{(r)}\right| \leq D^{-1} \sqrt{\frac{p}{2}}\left(\frac{1}{n-k} \sum_{1}^{n} c_{i}^{p}\right)^{1 / p} \sqrt{\operatorname{det} A A^{*}}
$$

where $D$ denotes the $G-C-D$ of all $k \times k$ determinants extracted from $A$.

Remark. The principal importance of such a generalization of Bombieri and Vaaler's result is that it takes into account, more strongly, the form of the matrix $A$. If the $c_{i}$ 's are all about the same size, then for $p>2$, the expression

$$
\left(\frac{1}{n-k} \sum c_{i}^{p}\right)^{1 / p}
$$

is small compared with the corresponding expression in which $p$ is replaced by 2 .

\section{REFERENCES}

[BF] I. Bárány and Z. Füredi, Computing the volume is difficult, Discrete Comput. Geom. 2 (1987), 319-326.

[BLM] J. Bourgain, J. Lindenstrauss and V. D. Milman, Approximation of zonoids by zonotopes, Acta Math. 162 (1989), 73-141.

[BP] K. M. Ball and A. Pajor, On the entropy of convex bodies with "few" extreme points, in preparation.

[BV] E. Bombieri and J. Vaaler, On Siegel's lemma, Invent. Math. 73 (1983), 11-32.

[CP] B. Carl and A. Pajor, Gelfand numbers of operators with values in a Hilbert space, Invent. Math. 94 (1988), 479-504.

[FJ] T. Fiegiel and W. B. Johnson, Large subspaces of $l_{\infty}^{n}$ and estimates of the Gordon-Lewis constants, Israel J. Math. 37 (1980), 92-112.

[G] E. D. Gluskin, Extremal properties of rectangular parallelipipeds and their applications to the geometry of Banach spaces, Mat. Sb. (N. S.) 136 (1988), 85-95.

[MeP] M. Meyer and A. Pajor, Sections of the unit ball of $l_{p}^{n}$, J. Funct. Anal. 80 (1988), 109-123.

[MiP] V. D. Milman and A. Pajor, Isotropic position and inertia ellipsoids and zonoids of the unit ball of a normed n-dimensional space, Israel seminar on G.A.F.A. (1987-88), Springer-Verlag, Lectures Notes in Math., vol. 1376, 1989.

[PT] A. Pajor and N. Tomczak-Jaegermann, Subspaces of small codimension of finite-dimensional Banach spaces, Proc. Amer. Math. Soc. 97 (1986), 637-642.

[S] C. Schütt, Entropy numbers of diagonal operators between symmetric Banach spaces, J. Approx. Theory 40 (1984), 121-128.

[V] J. D. Vaaler, A geometric inequality with applications to linear forms, Pacific J. Math. 83 (1979), 543-553.

Department of Mathematics, Texas A \& M University, College Station, Texas 77843

U. E. R. de Mathematiques, Université de Paris VII, 2 Place Jussieu, 75251 Paris Cedex 05, France 